\title{
PERSPECTIVAS EPISTEMOLÓGICAS EN LA FORMACIÓN DEL PROFESORADO DE EDUCACION FISICA: SUSTENTO TEÓRICO Y DIRECTRICES PARA LA PRÁCTICA EDUCATIVA
}

\section{PERSPECTIVES EPISTEMOLOGICAL IN THE FORMATION OF THE PROFESSORSHIP OF PHYSICAL EDUCATION: THEORETICAL SUSTENANCE AND DIRECTIVES FOR THE EDUCATIONAL PRACTICE}

\author{
Almonacid Fierro, Alejandro ${ }^{1} \&$ Moreno Doña, Alberto ${ }^{2}$ \\ ${ }^{1}$ Universidad Autónoma de Chile, sede Talca. \\ ${ }^{2}$ Universidad Austral de Chile.
}

ALMONACID F.A. \& MORENO D.A. Perspectivas Epistemológicas en la Formación del Profesorado de Educación Física: Sustento Teórico y Directrices para la Práctica Educativa. Mot. Hum. 13(2): 93-103; 2012.

\section{RESUMEN}

El presente artículo aborda la formación del profesorado en el área de Educación Física, en el bien entendido que éste es un proceso sistemático y organizado, tendiente a favorecer el desarrollo de los saberes propios del ejercicio profesional que se despliegan finalmente en el aula. La propuesta consiste en reflexionar acerca del sistema escolar como telón de fondo en el que se inscribe la acción educativa y las perspectivas paradigmático-epistemológicas en las que se sitúa la noción de aprendizaje en la disciplina. En este sentido se propone un paradigma formativo alejado de los clásicos esquemas deportivizados, más bien técnicos, y otros basados en la promoción de la salud, para avanzar hacia un modelo de formación de profesores de Educación Física desde una racionalidad crítica, eminentemente pedagógico y con una estructura valórica que favorece al desarrollo humano.

Palabras claves: paradigmas educativos, formación de profesores, educación física.

\begin{abstract}
The present article approaches the formation of the professorship in the area of Physical Education, in the understood that this one is a systematic and organized process, tending to favor the development of to know own of the professional exercise that is represented finally in the classroom. The offer consists of thinking brings over of the school system as backdrop in the one that registers the educational action and the perspectives paradigmatic - epistemological in that the notion of learning is located in the discipline. In this respect one proposes a formative paradigm removed from the classic sports schemes, rather technical, and different based on the promotion of the health, to advance towards a model of teachers' formation of Physical Education from a critical rationality, eminently pedagogic and with a structure of values that it favors to the human development.
\end{abstract}

Key words: educational paradigms, teachers' formation, physical education 


\section{INTRODUCCIÓN}

¿Cómo se ubicaban en el océano inmenso, profundo, travieso y misterioso, los primeros navegantes? ¿Cómo hicieron aquellos seres humanos que con entusiasmo, valentía y temor levantaron sus naves para deslizarse hacia lo desconocido? Ciertamente no lo sabemos, sólo contamos con ciertas aproximaciones que emergen fundamentalmente desde la imaginación, la duda y la incertidumbre.

Quizás los primeros navegantes se lanzaron a recorrer el mundo por la alfombra líquida de los mares a partir de su intuición, sin cartas, sin aparatos de medida, sin mapas, su único referente provenía de su sensibilidad para oler, escuchar y sentir el mundo que los rodeaba.

Algo había mas allá del horizonte que les quitaba el sueño, no les dejaba dormir, quizás eran gigantes, monstruos, precipicios que aparecían cada noche cual pesadilla para atormentarlos. En el fondo, por paradójico que parezca, se adentraban en lo desconocido huyendo de sí mismos y de sus permanentes desvelos.

Es probable que no fuera la razón, sino más bien la emoción, lo que los condujo a querer atrapar el horizonte $\mathrm{y}$, en consecuencia, descubrir nuevos y maravillosos mundos.

¿Por qué esta reflexión inicial? En este momento histórico la humanidad inicia un nuevo derrotero, un nuevo camino, una ruta no navegada, que precisa dejar de lado las certezas con que construimos los últimos tres siglos de nuestra era, vale decir, la historia de la época moderna, toda vez que el pensamiento filosófico y científico del siglo XVII consolida la racionalidad científica a partir de las teorías físicas de Newton, quien culmina el proyecto de la ciencia moderna. Por otro lado, y desde el punto de vista filosófico, Descartes situó el poder de la razón a nivel epistemológico, dejando de lado la perspectiva ontológica, de esta manera se consagra desde la filosofía la supremacía de la racionalidad científica y se adopta como modelo epistemológico la física-matemática. A partir de entonces la humanidad comienza un proceso de mudanza alejado de la emoción y la intuición, y comienza su derrotero hacia un mundo mecánico, racional y tecnológico $(1,2)$

En los albores del siglo XXI, parece ser que es hora de volver a re-encantarnos con las cuestiones fundamentales del vivir humano puesto que, durante más del noventa y nueve por ciento del transcurso de la historia humana, el mundo estuvo encantado y el hombre se veía a sí mismo como parte integral de él. Sólo recientemente, en los prolegómenos de la modernidad, cambiamos las coordenadas. Las cartas de navegación comenzaron a ser modificadas y re-estructuradas con nuevos códigos, reconfigurando los puntos cardinales iniciales. Necesitamos, por tanto, volver a reflexionar sobre nuestra propia humanidad, a la luz del contexto social y cultural del presente milenio.

\section{SOCIEDAD CONTEMPORÁNEA Y COMPLEJIDAD EDUCATIVA: LA NECESIDAD DE REFLEXIONAR SOBRE LA ESCUELA ACTUAL}

La complejidad e incertidumbre de los procesos socioculturales del mundo contemporáneo mantienen a la sociedad actual recorriendo sendas inimaginables hace sólo algunas décadas. Nuevas formas de ver y comprender la realidad han surgido como producto de nuevos descubrimientos científicos y tecnológicos, constituyendo un mundo digital y globalizado que ha llegado para quedarse.

La educación, como subsistema del sistema social, es impactada diaria y poderosamente por los cambios políticos, económicos, tecnológicos, sociales y culturales de la llamada postmodernidad. De esta manera, la macro configuración de la sociedad genera, en gran medida, las políticas y la estructura del sistema educativo imperante. En la práctica, se genera una potente y estrechísima relación de interdependencia, en la que la educación es influida por las demandas de la sociedad, y aquella influye, en el mediano y largo plazo, en las políticas educacionales de los países.

Los cambios que se producen en las sociedades son asumidos -consciente o inconscientemente, por el sistema educativo. Un ejemplo concreto de ello es el tipo de régimen de gobierno instalado; 
en una sociedad que transita de un sistema autoritario a uno democrático, valores como la participación, equidad, justicia social, el respeto a la diversidad, la tolerancia, y otros, se gestan, legitiman y expanden al interior del sistema educativo, conformando políticas e intervenciones educacionales que tienden al desarrollo de estos valores y a la revisión y superación de los propios de un sistema autoritario.

Chapman y Aspin (3), plantean la necesidad de realizar profundas transformaciones en los sistemas educativos actuales para que podamos enfrentarnos a los desafíos de la sociedad del conocimiento. En este contexto, el mundo occidental experimenta, desde hace algún tiempo, una profunda transformación, un cambio radical que afecta y afectará la forma de relacionarnos y, fundamentalmente, la forma en que aprendemos en un mundo globalizado. La década del noventa del siglo pasado, a partir de los significativos avances políticos y tecnológicos, determinó el nuevo rumbo de las sociedades y los significados asociados a ellas. Los mapas y códigos con los que navegábamos se volvieron obsoletos, surgieron nuevas constelaciones, nuevos faros, nuevos conocimientos, el mundo entró en una espiral de cambio e innovación insospechado.

Es así como la sociedad del conocimiento y la información irrumpen con fuerza arrolladora, desestabilizando los cimientos de todo lo construido, los avances científicos y tecnológicos se suceden día a día de manera exponencial, lo que antes nos llevó decenas de años hoy sólo depende de la velocidad tecnológica. Una de las características de la sociedad en que vivimos tiene que ver con el hecho de que el conocimiento es uno de los principales valores de sus ciudadanos, sin duda que esto golpea fuertemente las puertas de nuestros sistemas educacionales, conminándolos a un proceso de reflexión, análisis y autocrítica. La sociedad, por su parte, vuelve la mirada al sistema educacional intentando encontrar la respuesta de cómo enfrentar el futuro. Surgen innumerables preguntas en el espacio educativo: ¿cómo reaccionaremos a los cambios?, ¿cómo nos afectarán?, ¿cómo aprenderemos?, ¿a partir de qué valores nos relacionamos?, ¿es posible realizar un proceso significativo de mudanza?, entre otras posibles.
La educación continúa jugando el rol protagónico que nosotros -los seres humanos- le hemos encomendado, desde los tiempos en que el hombre se expresaba a través de símbolos y figuras en las paredes de las cavernas, hasta aquel que es capaz de adentrarse en el código genético para descubrir el maravilloso misterio de la vida y la creación. Por su parte, los sistemas educativos han tenido que asumir una responsabilidad fundamental en la conformación de las sociedades. En pleno siglo XXI deben entregar las orientaciones éticas y morales para profundizar hacia la infinitud del cosmos y, a la vez, preparar a los estudiantes para un mundo caracterizado por el individualismo, los fenómenos globales y un desarrollo científicotecnológico exponencial. Se esbozan, entonces, nuevos paradigmas epistemológicos y educativos que permiten una base de sustentación -algo más estable- para construir novedosos caminos, nuevas sendas y horizontes de desarrollo humano sustentable, tal y como lo expone Tedesco (4: pág. 57): "las instituciones educativas, los educadores, los intelectuales en general, ocuparán un lugar central en los conflictos y en las estrategias de intervención social y política".

De acuerdo a Morin (5, pág. 122) "la misión de la educación para la era planetaria es fortalecer las condiciones de posibilidad de la emergencia de una sociedad-mundo compuesta por ciudadanos protagonistas, conscientes y críticamente comprometidos en la construcción de una civilización planetaria”. Esta afirmación presenta una verdad ineludible. Se requiere de un nuevo sistema educativo que responda a las demandas de la sociedad del conocimiento y que a la vez favorezca el desarrollo de un sujeto que privilegia los aspectos más sentidos y profundos en los que exprese todo su potencial de humanidad. Revisemos a continuación algunas de las demandas y desafíos que se le presentan al sistema educativo contemporáneo desde esta perspectiva:

- Debiera ser inclusivo, vale decir, tener la capacidad de albergar a todos y a todas, no importando las aparentes diferencias. Ser capaz, entonces, de construir un currículo que acepte y respete la diversidad desde donde posibilitar la generación de nuevos saberes. 
- Los procesos de cambio-mudanza están incorporados en su estructura, de manera que es capaz de adaptarse rápidamente a nuevas concepciones.

- Énfasis en los procesos, y no solo en los resultados, lo que permite -entre otros elementos- re enfocar los modelos tradicionales de evaluación, para adecuarlos a la diversidad emergente de la sociedad.

- Privilegia el aprendizaje de los niños y niñas a partir del proceso de mediación pedagógica.

- Considerando los ingentes procesos de globalización, es capaz de rescatar el acervo cultural de los pueblos para otorgarle a la nueva generación, una identidad y singularidad que fortalezcan su idiosincrasia.

- La estructura valórica cruza transversalmente todo al quehacer de los niños y niñas, entregando un sustento ético $\mathrm{y}$ moral a sus actuaciones.

- Revaloriza a los niños y niñas a partir de la emoción más fundamental, el amor, tal y como lo propone Maturana (6, pág. 44), "si usted me pregunta cómo se aprende a amar, la respuesta es: en el vivir las acciones que constituyen al otro como un legitimo otro en la convivencia".

- En definitiva, un sistema educativo que rompe con los paradigmas tradicionales, para hacer del aprender a aprender, aprender a ser y el aprender a convivir, su centro neurálgico.

\section{EL CONOCER: UNA APROXIMACIÓN EPISTEMOLÓGICA DESDE LA TEORÍA DE LA COMPLEJIDAD}

Epistemología proviene del griego episteme, conocimiento o ciencia; y de logos, teoría o estudio. Significa, etimológicamente, 'ciencia del conocimiento', o 'teoría de la ciencia', y puede entenderse como la rama de la filosofía que estudia los problemas del conocimiento. En términos generales, se define como el análisis del conocimiento científico; en términos específicos analiza los supuestos filosóficos de las ciencias. Podemos reconocer, entonces, que esta 'ciencia del conocimiento' tiene un lugar protagónico y fundamental en uno de los espacios-tiempos donde se crea y desarrolla el conocimiento, vale decir, en la Escuela, entendida ésta como el centro de recreación del saber $(7,8,9)$

¿Entiende la Escuela que una de sus tareas fundamentales está relacionada con la re-creación y re-producción del conocimiento? La Escuela ha entendido que la división y la separación son acciones absolutamente normales que no merecen cuestionamiento e interpelación alguna, por tanto, para poder conocer es menester fraccionar, puesto que al conocer las partes, podemos comprender el todo. Se promueve, así, una relación sujeto-objeto a partir de la disociación (10), característica ésta representada, entre otros aspectos, por el llamado 'asignaturismo' escolar.

Ante esta situación, la propuesta urgente es construir un nuevo paradigma - quizás no tan nuevo, sino más bien el de siempre- que de cuenta de una epistemología concebida a partir de la unidad del conocimiento, en una concepción de carácter holística y hologramática en el que el todo está presente en las partes y las partes concurren en dicho todo. Es aquí donde cobra mucho sentido el llamado paradigma de la complejidad y la epistemología que de él se desprende. Ello nos permitirá comprender lo que está tejido conjuntamente. Un conocimiento que se enhebra, imbrica y se relaciona desde múltiples posibilidades $(11,10,12)$

La tarea de la institución escolar sería transitar desde un conocimiento superficial hacia un conocimiento complejo en el que no existe separación. No podría existir, en consecuencia, la clásica división entre teoría y practica, puesto que estas acciones son inseparables e indisociables para poder, precisamente, 'conocer' el fenómeno que nos ocupa. La actual disyunción entre mente y cuerpo responde, del mismo modo, al paradigma de la simplicidad, al entender que la razón o intelecto es algo distinto y separado del cuerpo, cuestión actualmente refutada por la moderna neurobiología. "Las representaciones que nuestro cerebro construye para describir una situación, y 
los movimientos formulados como respuesta a una situación, dependen de interacciones mutuas cerebro-cuerpo" (13, pág. 263).

\section{CONOCER, EPISTEMOLOGÍA EDUCACIÓN FÍSICA}

Adentrándonos ahora en nuestra área curricular, la Educación Física, nos preguntamos: ¿cuál es el cuerpo de conocimientos de la disciplina denominada Educación Física? Es una pregunta que genera cierta controversia entre los investigadores. Algunos se inclinan por proponer el fenómeno deportivo como esencia de la misma. Otros encuentran que la salud es el eje vertebrador. En una tercera opción están los que creen que la aptitud y la condición física serían el centro neurálgico de nuestra asignatura.

Para nosotros, la misma pregunta invita a una reflexión epistemológica, relacionada esta con el análisis y comprensión del conocimiento que emerge, se desarrolla y gestiona en la 'puesta en escena' de la Educación Física expresada en la acción.

Un problema que se presenta está relacionado con no poder definir, con claridad meridiana, cuál es el objeto de estudio de la Educación Física escolar. El nombre Educación Física explicita sólo un aspecto de la naturaleza humana, el referido al cuerpo o al físico, cuestión largamente estudiada desde el siglo XVII con los planteamientos de Rene Descartes y John Locke, quienes promovieron una mirada dualista del hombre y su educación, llegando incluso a formular que debido a que el sujeto estaba constituido por dos esencias fundamentales, era menester proveer dos tipos de educación distintas, una orientada a la razón o mente y otra dirigida al cuerpo. Desde esta mirada, el nombre Educación Física conduce a equivocación, y no nos permite precisar con certeza la naturaleza del conocimiento que esta disciplina desarrolla $(14,15,16)$

Desde el positivismo lógico, nuestro objeto de estudio sería el movimiento, expresado este en las distintas manifestaciones del deporte, el juego y la recreación, tanto individual como colectivamente, toda vez que los hechos observados vendrían a ser aquellos relacionados con técnicas deportivas, reglamentos, normas, rendimiento y competencia.
Lo observable y valorable se reduciría al movimiento, que es lo que aparentemente se visibiliza cuando analizamos la Educación Física desde su práctica escolar.

Gran parte de la investigación desarrollada da cuenta, precisamente, de este enfoque $(17,18,19$, 16), enfatizando cómo es la perspectiva que ha predominado en la formación inicial de profesores del área. Esta última idea será desarrollada a continuación, junto a los otros modelos de formación existentes.

\section{LA FORMACIÓN INICIAL EN EL ÁREA EDUCACIÓN FÍSICA}

En Chile, durante la década anterior, se celebraron 100 años de historia en el proceso de formación de profesores de Educación Física en el Instituto de Educación Física y Manualidades, creado en 1906, (20), lo que ha permitido que nuestro país cuente con una vasta experiencia en este ámbito, la que luego se ha esparcido a otras instituciones formadoras de profesores, de tal manera que en la actualidad existen más de 50 instituciones formadoras para el área que nos ocupa.

La formación de profesores, durante la primera parte del siglo $\mathrm{XX}$, tuvo un marcado acento militarista, producto de la influencia de las corrientes educativas provenientes principalmente de Alemania, las que acentuaban una práctica asociada a la disciplina y al deporte como medio para lograr el dominio del cuerpo. Lo que subyace en este paradigma es una noción de realidad, un pensamiento que considera al cuerpo como un objeto disociado de una mente que razona. En este contexto, más allá de una práctica fuertemente deportivizada que enfatiza y privilegia la dimensión biológica de la persona, lo que encontramos es una epistemología que asume el método científico, propio de la física newtoniana, como la manera válida para aproximarse al conocimiento. La disciplina se despliega fundamentalmente desde un hacer 'práctico' que se traduce en el desarrollo de ciertos estereotipos ligados a una técnica $\mathrm{y} / \mathrm{o}$ un movimiento específicos, como lo exponen Jaramillo, Portela y Murcia: 
"esta perspectiva de la formación invadió las entrañas de la Educación Física, de tal manera que todas las posibilidades de concebir el área se sumieron en prácticas que pregonaban, al igual que en la razón por un cuerpo bien formado, medidas hacia estereotipos que la cultura de los medios promociona; la búsqueda de índices elevados de rendimiento físico" (21, pág. 34)

Bajo este modelo paradigmático se han formado varias generaciones de profesores de Educación Física, los que finalmente reproducen en escuelas y liceos del país una visión de hombre que fragmenta $y$ reduce a la persona humana, poniendo el acento, casi de forma exclusiva, en ciertas dimensiones del hombre y no en una mirada de totalidad. Este modelo ha sido denominado, en la literatura pedagógica, como academicista o técnico.

\section{Modelo Academicista o Técnico.}

Es el modelo clásico. En él se concibe a los profesores como meros transmisores de un conocimiento técnico, medible y objetivable. Este modelo formativo está vinculado al positivismo lógico, buscando una verdad objetiva y la neutralidad científica. Prioriza los resultados y la eficacia docente en detrimento del proceso educativo. De acuerdo a Sancho y Ferrer, "se produce una estructura jerárquica y funcional, donde sus profesores están más preocupados por clasificar y ordenar a los estudiantes, que por detectar y superar los problemas de aprendizaje" (17, pág. 65)

El currículo es entendido como transmisión y prescripción, sometiendo la actividad docente a reglas técnicas que dictaminan cómo el profesorado debe resolver los problemas diarios, separando teoría y práctica, lo que genera una separación entre el trabajo teórico del 'experto' y la puesta en práctica del docente, tal y como lo expresa Magendzo

"el modelo racional-tecnológicopositivista-instrumentalista, postula que la planificación del currículo es un hacer técnico-científico, que posee una lógica lineal de pasos bien definidos (...) el modelo racional vuelve a aparecer en las nuevas tendencias para la elaboración del currículo, actualizado con el aporte de las teorías conductistas aplicadas a la enseñanza" (22, pág. 46)

Lo anterior se manifiesta en una clase de Educación Física que mantiene las clásicas polaridades y dualismos: mente-cuerpo, teoríapráctica, objeto-sujeto, causa-efecto, reflejados en una didáctica instructiva, frontal, medible, cuantificable y homogénea, que no respeta la diversidad e intereses de los niños. Este modelo recurre, en gran medida, al denominado diseño instruccional, caracterizado por tener un proceso de planificación 'riguroso', en el cual se definen, a partir del trabajo de 'expertos', todos los elementos de la acción educativa.

García (23, pág. 36) expone que "circunscribiéndonos al campo de la Educación Física, en el que la tradición investigadora se remonta a pocos años atrás, la perspectiva tradicionalista ha sido la predominante a lo largo de los años de existencia formal de nuestra disciplina" En este modelo el ideal educativo está orientado hacia la formación de un ser humano productivo, prestando especial atención a los elementos de carácter técnico, promoviendo el individualismo, el record, la marca y el modelamiento de gestos motores provenientes, fundamentalmente, del deporte de alto rendimiento.

En este mismo sentido, Kirk (24) plantea que, en la última parte del siglo $\mathrm{XX}$, los programas académicos de formación del profesorado de Educación Física se sustentaron -de manera importante- en las ciencias biofísicas del movimiento humano, lo que condujo a reinterpretar la disciplina y ubicarla en el ámbito de las ciencias de la actividad física. Ello con la intención de otorgarle el carácter científico a la disciplina, por lo que el paradigma epistemológico subyacente es de corte positivista, con la consiguiente utilización del método de la física clásica en la investigación del área.

Lo anterior resultó en currículos de formación del profesorado con un fuerte acento en las ciencias biológicas, en el bien entendido que la noción de 'cuerpo máquina' es la que subyace en esta 
perspectiva, toda vez que se privilegia el cuidado del 'cuerpo' como un dominio separado y distinto a los aspectos cognitivos, emocionales y/o relacionales de la persona. Desde el punto de vista metodológico-didáctico, la formación se redujo al aprendizaje y desarrollo de ciertos estereotipos dinámico-motrices ejecutados a través del mando directo $\mathrm{y}$, finalmente, a un proceso de evaluación instrumental, sumativa y con énfasis en el gesto motor $(25,17,26)$

\section{Modelo Práctico}

Como contrapunto a la mirada más bien academicista y técnica de la formación del profesorado en Educación Física, surge el enfoque desde la práctica. Según Castro, Correa y Lira (27, pág. 39), el interés de esta perspectiva "está dirigido a la comprensión de las situaciones humanas en interacción, pone énfasis en la deliberación ante las situaciones concretas de las practicas de enseñar y aprender, en lugar de centrarlo en los aspectos teóricos". Lo relevante en la comprensión del proceso de aprendizaje es la preocupación por la forma en que profesores y estudiantes construyen significados y le otorgan sentido a los aprendizajes (19)

La base del modelo es fenomenológica, entendiendo esta mirada como aquella que nos permite conocer los significados que los individuos le otorgan a su experiencia, intentando ver el mundo desde la perspectiva de otras personas, describiendo, comprendiendo e interpretándolo (28) En el plano educativo Sanjurjo (29, pág. 21) plantea que "desde esta concepción, la realidad escolar puede ser entendida a partir de los significados que le dan los sujetos (...), comprendiendo esas acciones en un contexto particular".

Curricularmente, este modelo formativo se focaliza en una racionalidad práctica que plantea las siguientes características (19)

- Hay un serio esfuerzo por superar las concepciones dualistas desde planteamientos de educación integral del ser humano. Existe, en consecuencia, un interés por la globalidad e interdisciplinariedad con el resto de las áreas y aprendizajes del alumnado, de tal manera que desde la Educación Física se genera la aspirada articulación de saberes.

- Surge el interés por desarrollar una Educación Física "para todas", inclusiva y comprensiva, permitiendo integrar a los más hábiles con los menos hábiles, favoreciendo y desarrollando la diversidad.

- Se concibe la Educación Física escolar como creación y recreación de cultura corpórea-motriz, en el bien entendido que las prácticas físico-deportivas poseen un carácter eminentemente antropológicocultural.

- Cobra especial importancia lo vivencial, la toma de conciencia, lo grupal, lo colaborativo y lo expresivo, superando el carácter individualista y competitivo de la Educación Física tradicional o tecnocrática.

- Existe una clara preocupación por los procesos de desarrollo personal y grupal, poniendo énfasis en los valores asociados a la democracia, libertad y participación.

- La metodología utilizada se centra en la experimentación, participación, búsqueda y descubrimiento; enfocada a la mejora de los aprendizajes y procesos.

- Se considera importante, y se fomenta, la participación del alumnado en la evaluación (en este sentido la autoevaluación y la evaluación compartida constituyen dos magníficas herramientas). La evaluación se entiende como un proceso de dialogo, participación y mejora.

- La base epistemológica está más cercana a las ciencias sociales y pedagógicas aplicadas al ámbito corpóreo-motriz.

- Se concibe al profesor de Educación Física como educador y como facilitador, orientador y generador de procesos de enseñanza-aprendizaje, coherente con la perspectiva del profesor investigadorreflexivo. 
ALMONACID F.A. \& MORENO D.A. Perspectivas Epistemológicas en la Formación del Profesorado de Educación Física: Sustento Teórico y Directrices para la Práctica Educativa. Mot. Hum. 13(2): 93-103; 2012.

En este proceso de construcción, la realidad escolar se va configurando a través de la articulación que hace el docente entre la acción que realiza y los marcos referenciales que posee en un proceso recursivo teórico-práctico y práctico-teórico, en el que la clásica distinción teoría y práctica es superada a través de la mirada holística y sistémica del fenómeno. Desde este modelo formativo, se produce un currículo de carácter práctico, abierto y flexible, favorecido por la actitud negociadora y deliberativa entre el profesor-alumnado, y donde éstos deben construir activamente sus propios aprendizajes.

\section{Modelo de Formación Crítico}

Este modelo emerge de la teoría crítica. Se caracteriza por una postura cuestionadora, problematizadora y reflexiva frente a la realidad social. La perspectiva crítica también atiende a la creación de significados, pero pone su eje en la potenciación de la emancipación de los seres humanos, concibiendo la enseñanza como praxis, entendida ésta como una actividad reflexiva que entrama acción y reflexión $(27,30,23)$ Podemos comprender, en consecuencia, que la teoría aporta niveles de análisis a la práctica y que en la misma práctica se producen procesos de reflexión sobre la acción, vale decir, un juego dialógico que va de la acción a la reflexión y de la reflexión a la acción. En este modelo el profesor es concebido como un investigador en el aula, reflexivo, crítico y comprometido con la Escuela y el contexto donde ésta se sitúa. El profesor es percibido como un intelectual, un agente de cambio social y político.

Desde la racionalidad crítica, tanto la práctica como la teoría son construcciones sociales que se llevan a cabo en contextos concretos. La enseñanza es entendida como una práctica socialmente construida, contextualizada sociohistóricamente, cargada de valores e intenciones que no puede analizarse desde la racionalidad técnica, sino desde los significados, condicionantes e intereses que la determinan (29, pág. 23). En palabras de Fraile,

"a partir de este modelo, la formación del profesorado de Educación Física se preocupa por generar estrategias docentes emancipatorias, reflexivas y críticas, que habitúan al estudiante a analizar las condiciones sobre las que actuará como docente; diseñando un trabajo colaborativo, donde el conocimiento se comparte desde un aprendizaje dialógico" (17, pág. 66).

Por su parte López y cols. (31) plantean que algunas de las cuestiones por resolver, desde una pedagogía crítica, en el ámbito de la Educación Física serían las siguientes:

- ¿Qué aporta la EF en la formación de seres humanos libres y autónomos?

- ¿Qué aporta la EF a la emancipación de la persona y los grupos sociales?

- ¿Qué aporta la EF a la construcción de un mundo más justo?

- ¿Qué puede aportar la EF en el desarrollo de los valores de libertad, igualdad y fraternidad?

- ¿Qué puede hacer la EF ante la agresión del medio natural?

- ¿Qué puede hacer la EF ante los trastornos alimentarios de adolescentes, jóvenes y adultos?

Para resolver estas cuestiones se requiere de una actitud autocritica, reflexiva e indagativa que favorezca el análisis de la propia práctica, en contexto, y el planteamiento de estrategias de mejora que favorezcan el desarrollo humano. Estos planteamientos transforman completamente la concepción del profesor de Educación Física, así como su formación inicial y permanente, acentuando de una manera consciente y crítica los procesos de investigación y reflexión personal sobre la propia práctica, sumados a procesos, hábitos y actitudes de trabajo cooperativo y de reflexión grupal.

Quizás es aquí donde se presentan los mayores desafíos para poder incorporar este paradigma en la formación del profesorado, puesto que la disciplina Educación Física responde a cuestiones más bien técnicas e instrumentales, toda vez que ha centrado su campo de actuación en el 
desarrollo del cuerpo o el físico, entendiéndose, en consecuencia, que la labor del profesor es más bien adiestrar, instruir, entrenar, y que su actuación depende de un grupo de profesionales expertos que diseñan y organizan el conocimiento que él debe reproducir, cuestión que se contrapone abiertamente con el modelo de formación crítico en el que se privilegian procesos participativos, democráticos y emancipatorios durante la formación del profesorado.

\section{ALGUNAS ORIENTACIONES PARA LA FORMACIÓN DE PROFESORES DE EDUCACIÓN FÍSICA: EL MODELO CRÍTICO COMO EJE VERTEBRADOR}

Las orientaciones que a continuación se presentan son un intento de poder concretar y operacionalizar algunos de los aspectos del modelo de formación crítico. Para ello hacemos una síntesis de lo propuesto por algunos autores que han trabajado en esta perspectiva $(32,18,33)$

- Prácticas formativas transdisciplinarias. Para ello es interesante que varias asignaturas puedan compartir tiempos y espacios pedagógicos, metodologías y estrategias evaluativas, de manera tal que se genere un diálogo fluido entre los saberes en función de un aprendizaje más significativo y pertinente.

- Aprendizaje colaborativo entre profesores/as, estudiantes, equipos de trabajo y grupo de clase. Dicho aprendizaje sustenta su existir en la intencionalidad de realizar una acción pedagógica que invite a desarrollar varias competencias relacionadas con la adquisición de principios éticos que precedan la práctica docente, la construcción de una actitud crítica ante los procesos de enseñanza y aprendizaje, y la observación analítica y reflexiva sobre la realidad de la Educación Física en el contexto escolar y social.

- Prácticas autoevaluativas. Argumentación apoyada en la toma de conciencia desde la reflexión del trabajo realizado, las metas alcanzadas, los compromisos cumplidos e incumplidos y, en general, de todo el proceso de formación $\mathrm{y}$ humanización desarrollado. Además de formativa, la evaluación también debe ser compartida, es decir, debe convertirse en un proceso democrático de diálogo y toma de decisiones colegiadas.

- El género como eje focalizador de las prácticas formativas. Los estudios dan cuenta de que si bien es posible observar una clara discriminación para la mayor parte de las niñas y para algunos niños, también es posible percatarse de algunas actitudes de resistencia al modelo deportivo tradicional, lógicas estas emergentes en las sociedades actuales, puesto que el enfoque de género ha permeado la practica educativa de los últimos 20 años.

- Educación Física y Salud. Si consideramos el concepto de salud como ausencia de enfermendad, conceptualización ésta muy común en la EF escolar, asumimos una serie de creencias encubiertas que nos hacen creer que la salud es algo individual y personal, responsabilizando a las personas de la buena o mala salud que estas puedan poseer. Se requiere, entonces, transitar hacia una perspectiva que aborda la problemática desde el sentido que la persona humana otorga a su vida $\mathrm{y}$, por consiguiente, a su corporalidad.

- Reinterpretación de los significados asociados al cuerpo, el ejercicio, el deporte, el sexo, la misma salud y los estilos de vida propios de la sociedad actual en donde nos desarrollamos. Es necesario entender que los problemas de salud, en coherencia con el apartado anterior, se contextualizan en los espacios $\mathrm{y}$ tiempos sociales $\mathrm{y}$ ambientales $\mathrm{y}$, por tanto, las estrategias que utilicemos en la educación física escolar para el trabajo de la salud deben ser a partir de una práctica física reflexionada y conceptualizada contextual y críticamente.

- La 'intervención' del profesor debe tener un carácter mediador y estar impregnada de entusiasmo, empatía y alegría, 
ALMONACID F.A. \& MORENO D.A. Perspectivas Epistemológicas en la Formación del Profesorado de Educación Física: Sustento Teórico y Directrices para la Práctica Educativa. Mot. Hum. 13(2): 93-103; 2012.

buscando transformar el acto educativo cotidiano en una aventura que invite a descubrir nuevos significados para un mundo en cambio.

- Relación afectiva entre docentes y discentes, producto de una coherencia y consistencia íntima que considera al alumno como un legítimo otro, como un proyecto inacabado de unicidad y a quien se debe escuchar $y$ atender en su individualidad. El respeto a la diversidad es un principio que el profesor debe hacer suyo para enfrentar los procesos educativos-formativos con posibilidades de desarrollo real.

- El profesor debe centrar el proceso educativo en el aprendizaje de los alumnos, cuestión que conlleva cambiar el paradigma tradicional predominantemente centrado en la enseñanza, por aquel que pone énfasis en el auténtico aprendizaje de los estudiantes, con la consiguiente generación de conocimientos a partir de las historias y vivencias de cada uno de ellos.

En definitiva, podemos plantear que los desafíos para el profesor de Educación Física del presente, se traducen -primero- en una noción ontológica de la persona humana como sujeto primero y último de la acción educativa, seguidamente de una concepción epistemológica que favorece la articulación de saberes, el sentido y significado de una práctica corpórea-motriz $\mathrm{y}$, por último, el conocimiento y transformación del contexto donde se generan las prácticas educativas.

\section{A modo de cierre}

De acuerdo a lo anteriormente expuesto, se desprende que la formación del profesorado en el área de Educación Física se concibe y despliega desde diferentes perspectivas epistemológicas. Desde nuestra postura se debiera aspirar a un modelo de formación de profesores siempre en construcción, con énfasis en el aprendizaje de los estudiantes, que promueva un cambio profundo en la práctica docente caracterizada por la implicancia íntima y honesta de los alumnos en sus procesos formativos.
Se necesita de un cambio decidido y riguroso en la formación y en el rol de los profesores de Educación Física, redefiniéndose como mediadores, animadores y facilitadores de los procesos de aprendizaje de sus estudiantes. El docente debiera partir de principios educativos que contemplen valores de carácter democratizador, como el respeto, la tolerancia y la colaboración. Todo ello en pos de promover y favorecer el desarrollo humano.

En definitiva, un modelo de formación que busca la mejora del sistema en su conjunto, a partir de un respeto irrenunciable por la persona y que aborda, desde un nueva concepción epistemológica, los procesos de aprender.

\section{REFERENCIAS BIBLIOGRÁFICAS}

1. Berman, M. El reencantamiento del mundo. Santiago de Chile: Cuatro Vientos. 2004.

2. Echeverría, R. El Búho de Minerva. Santiago de Chile. JC Sáez Editor. 1997.

3. Chapman, J., y Aspin, D. (2001). Schools and the Learning Community: Laying the Basis for Learning Across the Lifespan. En D. Aspin, J. Chapman, M. Hatton e Y. Sawano (eds.). International Handbook of Lifelong Learning. London: Kluwer, pp. 405-446.

4. Tedesco, J. Educar en la sociedad del conocimiento. Buenos Aires: Fondo de cultura económica. 2005 .

5. Morin, E. Educar en la era planetaria. Barcelona: Gedisa. 2003

6. Maturana, H. El sentido de lo humano. Santiago de Chile: Lom. 2005.

7. Briones, G. Epistemología de las ciencias sociales. Bogota: ICFES. 1996.

8. Freire, A. Retamal, J. Sepúlveda, R. La aventura epistemológica de nuestra propia comprensión, una mirada desde la pedagogía. Santiago: Ediciones UCSH. 2003.

9. Osorio, F. Epistemología de las ciencias humanas. Santiago de Chile: Ediciones Universidad Católica Silva Henriquez. 2007.

10. Morin, E. Los siete saberes necesarios para la educación del futuro. Barcelona: Paidós. 2001.

11. Morin, E. El método III. El conocimiento del conocimiento. Madrid: Cátedra. 1994.

12. Morin, E. Introducción al pensamiento complejo. Barcelona: Gedisa. 2005.

13. Damasio, A. El error de Descartes. Barcelona: Crítica. 2001. 
ALMONACID F.A. \& MORENO D.A. Perspectivas Epistemológicas en la Formación del Profesorado de Educación Física: Sustento Teórico y Directrices para la Práctica Educativa. Mot. Hum. 13(2): 93-103; 2012.

14. Almonacid, A. Jugando con el trío complejidad, epistemología, motricidad desde la educación física. En Revista Pensamiento Educativo, 2006: 38, 141-153.

15. Toro, S. Una aproximación epistemológica a la didáctica de la motricidad desde el discurso y práctica docente. En Revista Estudios Pedagógicos, 2007, 37, 1, 29 43 .

16. Moreno, A. Percepciones del profesorado universitario en relación a la función de transformación social de la educación física como asignatura del currículum escolar: el caso de Chile. Granada: Universidad de Granada. 2011.

17. Fraile, A. Nuevos Caminos para la Formación del Profesorado de Educación Fisica. En Revista de Ciencias del Deporte, Educación Física y Recreación, Kinesis, 2003, 36, $62-71$.

18. Rivera, E.; Trigueros, C.; De La Torre, E. y Moreno, A. Formar docentes, formar personas: una experiencia transdisciplinar para democratizar el aula universitaria. En L'Activitat del Docent: Intervenció, Innovació, Investigació., 2010, Barcelona: CiDd: II Congrés Internaiconal de Didáctiques. Págs.: 1-8.

19. López, V. Monjas, R. Pérez, D. Buscando alternativas a la forma de entender y practicar la educación física escolar. Zaragoza: INDE. 2003.

20. Soto, F. El Estado y el Instituto Pedagógico, dos historias, un norte. En Revista Intramuros, 10. 2002.

21. Jaramillo, L. Portela, H. Murcia, N. La educación física: ¿un problema de preparación o seducción? Armenia: Kínesis. 2005.

22. Magendzo, A. Dilemas del currículo y la pedagogía. Santiago de Chile: Lom. 2008.

23. Garcia, H. La formación del profesorado de educación física: problemas y expectativas. Barcelona: INDE. 1997.

\section{Dirigir correspondencia a:}

Alejandro Almonacid Fierro aalmonacidf@uautonoma.cl 5 Poniente, 1670

Talca - Chile

\section{RECIBIDO: 7-11-2012}

ACEPTADO: 17-12-2012
24. Kirk, D. Fundamentos para una pedagogía crítica en la formación del profesorado de Educación Física. En J. Devis (coord.). La educación física, el deporte y la salud en el siglo XXI. Alcoy: Marfil. 2001. Págs.: 101-107.

25. Camacho, H. Pedagogía y didáctica de la Educación Física. Armenia: Kinésis. 2003.

26. Almonacid, A. (2010). Aproximación a un estado del arte en la formación de profesores de Educación Física. Puntuaciones y reflexiones posibles. En Revista Motricidad y Persona, 2010, 7, 17 -25.

27. Castro, F. Correa, M. y Lira, H. Currículo y evaluación educacional. Hualpen: Ediciones Universidad del Bio - Bio. 2006.

28. Rodríguez, G. Gil, J. y García, E. Metodología de la investigación cualitativa. Málaga: Ediciones Aljibe. 1996.

29. Sanjurjo, L. La formación práctica de los docentes, reflexión y acción en el aula. Rosario - Santa Fe: HomoSapiens. 2002.

30. Freire, P. Pedagogía del oprimido. Buenos Aires: Siglo XXI. 2005.

31. López, V. y Cols. Algunas reflexiones sobre Educación Física y pedagogía crítica. En Revista RETOS. Nuevas tendencias en Educación Física, deporte y recreación, 2002, 2, 30-35.

32. Rivera, E; de la Torre, E. Democratizar el aula universitaria: una propuesta alternativa de formación inicial universitaria desde la participación del alumnado. En Investigación en la Escuela, 2005, 57, 85-95.

33. Soler, S. Los procesos de reproducción, resistencia y cambio de las relaciones tradicionales de género en la Educación Física: el caso del fútbol. Cultura y Educación, 2009,

21 ,

1 ,

$31-42$. 